\begin{tabular}{|c|c|}
\hline & $\begin{array}{c}\text { XV Encontro Nacional de Tecnologia do } \\
\text { Ambiente Construído }\end{array}$ \\
\hline & 12.13 14 DE NOVEMBR \\
\hline
\end{tabular}

\title{
DESEMPENHO DE EMPREENDIMENTOS HABITACIONAIS: UMA ANÁLISE COMPARATIVA DOS CRITÉRIOS CONTIDOS NA NBR 15575 E NO REFERENCIAL AQUA
}

OLIVEIRA, Vivian Moreno (1). HIPPERT, Maria Aparecida Steinherz (2)

(1) Universidade Federal de Juiz de Fora, e-mail: vivian.moreno@ hotmail.com (2) UFJF, e-mail: aparecida.hippert@ufjf.edu.br

\begin{abstract}
RESUMO
A produção de habitações no Brasil, em larga escala e sem parâmetros mínimos de desempenho resultou, durante anos, na construção de moradias precárias com qualidade bastante inferior às necessidades dos usuários. Diante deste histórico de baixa qualidade das habitações surge a necessidade de estabelecer o desempenho mínimo para a construção das novas moradias através da norma ABNT NBR 15575:2013 Edificações habitacionais - Desempenho. Com a norma em vigor, torna-se imprescindível que os conceitos de desempenho também sejam contemplados pelos sistemas de certificação, uma vez que a maioria deles está orientado a uma avaliação prescritiva. O objetivo desta pesquisa é identificar se algum sistema de certificação ambiental, a exemplo do referencial AQUA já está atendendo às exigências de desempenho conforme a norma brasileira de desempenho. $\mathrm{O}$ método de pesquisa adotado é uma revisão bibliográfica de cunho descritivo e exploratório. Para isto será realizada uma análise comparativa entre os critérios estabelecidos no Referencial Técnico de Alta Qualidade Ambiental (AQUA) para Edifícios Habitacionais e aqueles estabelecidos na norma ABNT NBR 15575:2013. Os resultados das análises identificaram que alguns critérios de desempenho contidos na referida norma já estão sendo atendidos pelo referencial AQUA. Mesmo diante das dificuldades para aplicação da norma, as exigências de desempenho já estão sendo incorporadas, ainda que aos poucos, na forma de projetar e construir no Brasil.
\end{abstract}

Palavras-chave: Desempenho de edificações, NBR 15575, referencial AQUA e critérios de desempenho.

\begin{abstract}
The production of housing in Brazil, on a large scale and without minimum performance parameters has resulted, for years, building makeshift quite inferior quality to user needs. Given this historical low quality of housing is the need to establish minimum performance for the construction of new housing through ABNT NBR 15575:2013 - Residential Buildings - Performance. With the rules in force, it is essential that the concepts of performance are also covered by certification schemes, since most of them are oriented to a prescriptive evaluation. The objective of this research is to identify whether any environmental certification system, such as the reference AQUA is already meeting the performance requirements according to the Brazilian standard of performance. The research method adopted is a literature review of descriptive and exploratory nature. For this a comparative analysis of the criteria established in the Technical Reference High Environmental Quality (AQUA) for Housing buildings will be held and those of the Standard ABNT NBR 15575:2013 will be held. The results of the analyzes identified some performance indicators contained in that rule are already being met by AQUA. Even in the face of difficulties for application of the standard, the requirements are already being incorporated, albeit slowly, in the form of design and build in Brazil.
\end{abstract}

Keywords: Performance of buildings, NBR 15575, AQUA framework and performance criteria 


\section{INTRODUÇÃO}

O Brasil produz muitas habitações, cuja produção é quase artesanal e mais onerosa com qualidade inferior àquela desejável demandando de parâmetros mínimos para garantir uma habitação adequada (IPT, 1998). No Brasil, ainda existe o desafio em produzir habitações em quantidade que vá diminuindo o déficit, com as premissas do preço adequado e da qualidade que possam corresponder à realidade socioeconômica do país (ROQUE, 2009). Diante do crescimento do mercado e da baixa qualidade dos empreendimentos habitacionais é necessário discutir qual o desempenho mínimo para as novas moradias a serem construídas (BORGES e SABBATINI, 2008).

Atualmente, os conceitos de sustentabilidade na construção civil vêm sendo aplicados por meio dos sistemas de certificação ambiental. No entanto, estão orientados para uma avaliação prescritiva e traduzem, ainda pouco relevante em seus métodos de avaliação, os impactos ao longo do ciclo de vida da edificação (SILVA, 2007). Neste sentido, a autora discute a importância da integração dos conceitos de desempenho nos sistemas de avaliação, uma vez que a maioria dos métodos desenvolvidos está orientada a uma avaliação ambiental e prescritiva. O desenvolvimento ideal das metodologias de avaliação de edifícios é migrar dos critérios prescritivos para critérios de desempenho.

Sendo assim, o objetivo deste trabalho é identificar se o sistema de certificação ambiental, a exemplo do AQUA já atende às exigências de desempenho estabelecidas na norma NBR 15575. Os critérios de desempenho ambiental analisados serão aqueles estabelecidos no Referencial Técnico de Alta Qualidade Ambiental (AQUA) para Edifícios Habitacionais, por ser um sistema de avaliação ambiental internacional com adaptações voltadas para as características brasileiras de desenvolvimento sustentável.

\section{O CONCEITO DE DESEMPENHO NA CONSTRUÇÃO CIVIL}

A construção civil vem sendo impulsionada pelas inovações das tecnologias construtivas demandando novos materiais, técnicas e sistemas construtivos. Neste sentido, a construção vem se tornando cada vez mais industrializada com sistemas construtivos que garantem maior agilidade na execução das edificações. Desta necessidade de industrialização da construção civil surgiu a consciência da avaliação de desempenho e do controle da qualidade na produção dos edifícios habitacionais (MITIDIERI FILHO e HELENE, 1998).

No Brasil, até a década de 80 o conceito de desempenho estava voltado para os aspetos conceituais do termo associado a um nível de desempenho desejado, ou seja, das expectativas do usuário em relação ao produto comparado ao desempenho do produto entregue. A partir da década de 90 o conceito de desempenho foi impulsionado principalmente pelas questões de sustentabilidade sendo aplicado o conceito de desempenho na concepção e execução das construções habitacionais (ROQUE, 2009).

O conceito de desempenho na construção civil está relacionado principalmente ao atendimento das exigências e necessidades dos usuários, mas também está associado ao termo durabilidade, na qual foram introduzidos importantes conceitos, como vida útil de projeto (VUP) e prazos de garantia, segundo Oliveira e Mitidieri (2012). Através destas exigências é possível estimar o desempenho potencial a partir de uma combinação de métodos de avaliação para avaliar o produto prevendo seu comportamento, quando em utilização normal (MITIDIERI FILHO e HELENE, 1998). Portanto, a avaliação do desempenho permite verificar se o edifício e suas partes atendem às condições estabelecidas. 


\section{$3 \quad$ ESTRUTURA DA NBR 15575}

A norma brasileira de desempenho é composta por seis partes. A primeira parte apresenta os requisitos gerais que se aplicam às edificações habitacionais, como um todo integrado. As demais partes da norma correspondem aos sistemas que compreendem uma edificação, a saber: sistemas estruturais, sistemas de pisos, sistemas de vedações verticais internas e externas, sistemas de coberturas e sistemas hidrossanitários.

A norma de desempenho foi pautada em requisitos estabelecidos pelas normas ISO 6240, de 1980 e ISO 6241, de 1984 e baseada na tradução das necessidades dos usuários (BORGES e SABBATINI, 2008). Através da revisão das normas internacionais, foi possível estabelecer as necessidades para assegurar o desempenho conforme as expectativas dos usuários. $\mathrm{Na}$ estrutura da norma foram definidas doze exigências apresentadas no Quadro 1.

Quadro 1 - Exigências dos usuários da NBR 15575

\begin{tabular}{|l|l|}
\hline SEGURANÇA & $\begin{array}{l}\text { Desempenho estrutural } \\
\text { Segurança contra incêndio } \\
\text { Segurança no uso e na operação }\end{array}$ \\
\hline \multirow{3}{*}{ HABITABILIDADE } & Estanqueidade \\
& Desempenho térmico \\
& Desempenho acústico \\
& Desempenho lumínico \\
& Saúde, higiene e qualidade do ar \\
& Funcionalidade e acessibilidade \\
& Conforto táctill, visual e antropodinâmico \\
\hline SUSTENTABILIDADE & Durabilidade e manutenibilidade \\
& Impacto ambiental \\
\hline
\end{tabular}

Fonte: Adaptado de NBR 15575-1 (ABNT, 2013).

Todas as partes da norma contemplam as doze exigências descritas na primeira parte da norma de desempenho, porém cada uma terá o foco voltado para o sistema a que se refere com requisitos e critérios característicos a cada um. Além disto, são estabelecidos os métodos de avaliação e níveis de desempenho para cada sistema, componente ou elemento analisado.

Os requisitos especificam os níveis mínimos de desempenho que devem ser alcançados para garantia da vida útil e desempenho do sistema avaliado. Os critérios de desempenho expressam a quantificação dos requisitos abordando as exigências dos usuários e as condições de exposição a que está submetida à edificação. Os métodos de avaliação especificam a forma de avaliação para certificação de desempenho de um determinado subsistema e podem ser estabelecidos através de análises visuais e de análise de projeto arquitetônico, de ensaios in loco, cálculos ou ensaios em laboratórios.

Por fim, são descritos os níveis de desempenho que estabelecem os valores mínimos de desempenho que devem ser considerados para atender satisfatoriamente ao método de avaliação aplicado. Sendo assim, atendendo aos requisitos e critérios de acordo com os métodos de avaliação e níveis de desempenho especificados para cada sistema, considera-se que as exigências dos usuários foram satisfatoriamente atendidas. 


\section{ESTRUTURA DO REFERENCIAL AQUA}

A metodologia para certificação no processo AQUA se dá através do atendimento aos indicadores de sustentabilidade garantindo o nível de desempenho mínimo em todas as categorias. Para isto requer que o empreendedor adote um Sistema de Gestão de Empreendimento (SGE) e atenda as exigências necessárias para alcançar a Qualidade Ambiental (QAE). A QAE é estabelecida por 14 categorias e se reúnem em quatro famílias: (1) Sítio e Construção, (2) Gestão, (3) Conforto e (4) Saúde, conforme apresentadas no Quadro 2.

Quadro 2 - Categorias AQUA

\begin{tabular}{|c|c|}
\hline SÍTIO E CONSTRUÇÃO & CONFORTO \\
\hline $\begin{array}{l}1 \text { - Relação do edifício com o entorno } \\
2 \text { - Escolha integrada de produtos, sistemas, e } \\
\text { processos construtivos } \\
3 \text { - Canteiro de obras com baixo impacto } \\
\text { ambiental }\end{array}$ & $\begin{array}{l}8 \text { - Conforto higrotérmico } \\
9 \text { - Conforto acústico } \\
10 \text { - Conforto visual } \\
11 \text { - Conforto olfativo }\end{array}$ \\
\hline GESTÃO & SAÚDE \\
\hline $\begin{array}{l}4 \text { - Gestão da energia } \\
5 \text { - Gestão da água } \\
6 \text { - Gestão dos resíduos de uso e operação do } \\
\text { edifício } \\
7 \text { - Gestão da manutenção }\end{array}$ & $\begin{array}{l}12 \text { - Qualidade sanitária dos ambientes } \\
13 \text { - Qualidade sanitária do ar } \\
14 \text { - Qualidade sanitária da água }\end{array}$ \\
\hline
\end{tabular}

Fonte: FCAV, 2013.

Na estrutura AQUA, as categorias são desmembradas em subcategorias e depois são desmembradas em exigências e comentários. As categorias representam os desafios ambientais de um edifício, sendo aplicado a um empreendimento novo ou reabilitado. As subcategorias expressam as principais preocupações associadas a cada desafio ambiental. Cada preocupação estabelece as exigências que são definidas através de ações e indicadores (parâmetros ou critérios) de desempenho ambiental para seu atendimento.

No referencial, as exigências são acompanhadas das especificações e dos critérios prescritivos ou de desempenho e deverão ser baseadas em elementos qualitativos através de especificações, elementos gráficos, estudos - ou quantitativos por meio de métodos de avaliação, softwares, memória de cálculo, etc. A avaliação da QAE consiste em verificar se as características do empreendimento atendem a certos critérios, identificando o nível de desempenho atingido (BARATELLA, 2011). Para aplicação do referencial, o empreendedor deverá buscar o melhor desempenho sempre que possível.

\section{METODOLOGIA}

O método de pesquisa adotado é uma revisão bibliográfica de cunho descritivo e exploratório. A estrutura original do referencial AQUA e da norma de desempenho foram reorganizadas para facilitar a análise comparativa. Para isto, haverá a compatibilização dos termos e nomenclaturas, sem alteração do conteúdo e das informações características a cada uma das referências analisadas. A estrutura elaborada está representada na Figura 1. 
Figura 1 - Proposta de estruturação da NBR 15575 e do referencial AQUA

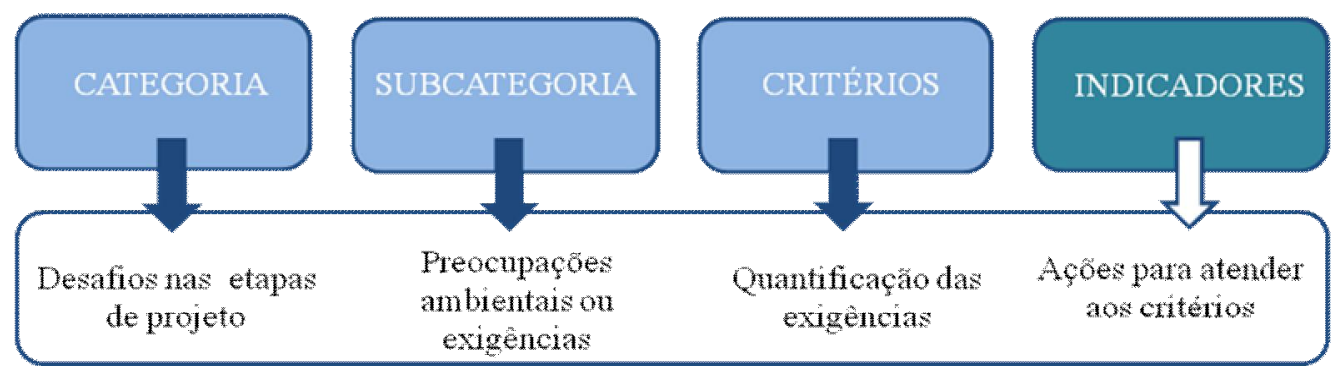

Fonte: Elaborado pelas autoras.

O termo CATEGORIA definirá as etapas que compreendem o processo de projeto. Estas etapas foram definidas em três categorias: PROJETO, CONFORTO E GESTÃO. A etapa de conforto, embora seja realizada na etapa de projeto foi separada por apresentar variados temas, ou seja, a temática é tão extensa quanto à etapa de projeto. $\mathrm{O}$ referencial AQUA apresenta sua estrutura já dividida em categorias, que representam os desafios ambientais. As SUBCATEGORIAS correspondem às principais preocupações associadas a cada desafio ambiental. Na NBR 15575, as subcategorias correspondem às exigências dos usuários. Após a definição das categorias, o mesmo processo de compatibilização dos termos foi realizado para as subcategorias. Esta padronização será fundamental para facilitar a análise comparativa.

Em etapa posterior, as subcategorias foram desmembradas em CRITÉRIOS, que estabelecem exigências visando à sustentabilidade, no caso do AQUA ou das exigências dos usuários, no caso da NBR 15575. Na norma, os critérios estão bem definidos, já que esta nomenclatura parte da sua estrutura original. O referencial AQUA, no entanto, estabelece uma lista denominada comentários-exigências, onde estão inseridas as exigências visando à sustentabilidade. Por isto, coube o exercício de desassociar entre os comentários e exigências o que seria exigido como um critério e o que seriam as ações para atendimento dos critérios.

Por fim, foram identificados os INDICADORES ambientais ou de desempenho, ou seja, as ações a serem adotadas para atender os critérios. Os indicadores apontam soluções projetuais através de métodos prescritivos ou por meio de métodos de simulação e de desempenho.

A partir da compatibilização dos termos e nomenclaturas, o referencial AQUA e todas as partes da norma foram reorganizadas conforme esta nova estrutura. Para análise comparativa, serão avaliadas primeiramente as subcategorias existentes na norma de desempenho que são contempladas pelo AQUA. Após identificar as subcategorias em comum será possível comparar os critérios exigidos no referencial com aqueles definidos na norma de desempenho e quantificá-los, obtendo os resultados finais.

\section{RESULTADOS E ANÁLISES}

O Quadro 3 apresenta as categorias e subcategorias identificadas na reorganização da estrutura para análise comparativa. As subcategorias do AQUA são classificadas conforme o potencial de atendimento aos critérios e indicadores de desempenho exigidos pela NBR 15575. Neste caso, são definidos três critérios para análise: o não atendimento aos critérios da norma (NÃO), o atendimento as exigências estabelecidas pela norma (SIM) e o atendimento parcial (PARCIAL). O atendimento parcial pode corresponder ao fato do sistema de certificação apresentar o mesmo tema da 
subcategoria e não apresentar critérios similares aos exigidos na norma. E ainda, apresentar o mesmo critério, porém com indicadores diferentes.

Quadro 3 - Categorias e subcategorias do referencial AQUA e da NBR 15575

\begin{tabular}{|c|c|c|}
\hline CATEGORIA/SUBCATEGORIA & AQUA & NBR 15575 \\
\hline \multicolumn{3}{|l|}{ PROJETO } \\
\hline Implantação do empreendimento & Sim & Sim \\
\hline Escolha dos sistemas e processos construtivos & Sim & Sim \\
\hline Qualidade sanitária dos ambientes & Sim & Sim \\
\hline Qualidade sanitária do ar & Parcial & Sim \\
\hline Qualidade sanitária da água & Parcial & Sim \\
\hline Funcionalidade e acessibilidade & Parcial & Sim \\
\hline Desempenho estrutural & Não & Sim \\
\hline Segurança no uso e na operação & Parcial & Sim \\
\hline Segurança contra incêndio & Não & Sim \\
\hline Estanqueidade & Parcial & Sim \\
\hline Durabilidade & Parcial & Sim \\
\hline \multicolumn{3}{|l|}{ CONFORTO TÉRMICO } \\
\hline Eficiência da envoltória & Sim & Sim \\
\hline Ventilação natural & Sim & Sim \\
\hline Desempenho no verão & Não & Sim \\
\hline Desempenho no inverno & Sim & Sim \\
\hline \multicolumn{3}{|l|}{ CONFORTO LUMÍNICO } \\
\hline Iluminação natural & Sim & Sim \\
\hline Iluminação artificial & Sim & Sim \\
\hline \multicolumn{3}{|l|}{ CONFORTO ACÚSTICO } \\
\hline Ruídos de impacto & Sim & Sim \\
\hline Isolamento dos ruídos entre UH's & Sim & Sim \\
\hline Isolamento acústico de vedações & Sim & Sim \\
\hline Ruídos em equipamentos prediais & Não & Sim \\
\hline \multicolumn{3}{|l|}{$\begin{array}{l}\text { CONFORTO TÁTIL E } \\
\text { ANTROPODINÂMICO }\end{array}$} \\
\hline Conforto tátil e adaptação ergonômica & Não & Sim \\
\hline Adequação antropodinâmica & Não & Sim \\
\hline Homogeneidade e planeza dos acabamentos & Não & Sim \\
\hline Conforto na operação dos sistemas prediais & Não & Sim \\
\hline \multicolumn{3}{|l|}{ GESTÃO DA ENERGIA } \\
\hline Dispositivos economizadores & Sim & Sim \\
\hline \multicolumn{3}{|l|}{ GESTÃO DA ÁGUA } \\
\hline Dispositivos economizadores & Parcial & Sim \\
\hline \multicolumn{3}{|l|}{ GESTÃO DA MANUTENÇÃO } \\
\hline Manutenibilidade do edifício e seus sistemas & Sim & Sim \\
\hline Resistência ao desgaste em uso & Não & Sim \\
\hline \multicolumn{3}{|l|}{ GESTÃO DOS RESÍDUOS } \\
\hline Gerenciamento de resíduos & Sim & Sim \\
\hline
\end{tabular}

Fonte: Elaborado pelas autoras. 
Na categoria Projeto, o referencial AQUA e a norma de desempenho apresentam nove subcategorias em comum. A norma estabelece recomendações para a Implantação do empreendimento considerando os riscos de deslizamentos de taludes, enchentes, erosões, assoreamentos de vales ou cursos d'água. Para isto, faz referência a outras normas, bem como a legislação vigente do local. O referencial também apresenta exigências tomadas em relação à topografia e características do solo do terreno através de soluções projetuais que não estão pautadas em normas ou legislações, ou seja, os indicadores são prescritivos.

Na subcategoria Escolha dos sistemas e processos construtivos, a norma estabelece recomendações quanto ao uso de madeira certificada, uso de materiais com baixo impacto ambiental e a implementação da gestão de resíduos no canteiro de obra que são contempladas em vários outros critérios do referencial. Neste sentido, ambos definem os mesmos indicadores para atender a exigência de forma prescritiva.

Na subcategoria Qualidade sanitária dos ambientes, a norma apresenta um único critério que é atendido pelo referencial AQUA. O referencial atende aos mesmos indicadores exigidos pela norma NBR 15575 e tratam de soluções projetuais que atendam a legislação vigente. Na subcategoria Qualidade sanitária do ar, a norma de desempenho estabelece quatro critérios. Nesta subcategoria, o AQUA atende parcialmente à norma de desempenho, uma vez que são atendidos dois critérios dentre os quatro exigidos pela norma NBR 15575.

A norma de desempenho apresenta quatro critérios na subcategoria Qualidade sanitária da água. Os critérios estabelecidos pelo referencial AQUA não são os mesmos exigidos pela norma. Sendo assim, o referencial AQUA atende parcialmente a esta subcategoria, uma vez que já adota alguns critérios sobre a temática, embora não atendam aqueles exigidos pela norma. Os critérios em comum estabelecidos na subcategoria Funcionalidade e acessibilidade são atendidos pelo referencial com indicadores similares aos exigidos na norma. Ambos estabelecem indicadores a serem atendidos em projeto. No entanto, o referencial AQUA atende a apenas três, dos dez critérios exigidos pela norma NBR 15575.

Na subcategoria Segurança no uso e na operação, a norma de desempenho estabelece vinte critérios, sendo que apenas um deles é atendido pelo referencial AQUA. Ainda que o referencial AQUA atenda aos mesmos indicadores exigidos pela norma, outros critérios ainda não são contemplados. Portanto, esta subcategoria é atendida parcialmente. A subcategoria Estanqueidade é característica da norma de desempenho, que estabelece dezessete critérios, onde somente um deles é atendido pelo referencial AQUA. Ainda que o AQUA atenda a apenas um critério, esta subcategoria é atendida parcialmente, já que atende a temática exigida pela norma.

$\mathrm{Na}$ subcategoria Durabilidade, dos quatorze critérios estabelecidos pela norma de desempenho, quatro são atendidos pelo sistema de certificação. O AQUA atende aos critérios relativos apenas aos sistemas de pisos. Os critérios exigidos na norma para os demais sistemas não são contemplados pelo AQUA. Sendo assim, para efeito da análise comparativa entende-se que esta subcategoria é parcialmente atendida pelo AQUA.

$\mathrm{Na}$ categoria Conforto térmico foram identificadas quatro subcategorias comuns a norma de desempenho e ao referencial AQUA. Em todas as subcategorias, o referencial AQUA apresenta os mesmos indicadores para os critérios em comum com a norma de desempenho, seja referenciando a norma ou exigindo os mesmos métodos de avaliação que são exigidos pela norma NBR 15575. Sendo assim, esta subcategoria é atendida pelo sistema de certificação analisado. 
A categoria Conforto lumínico apresenta duas subcategorias contempladas pelo sistema de certificação e pela norma. Dos três critérios exigidos pela norma de desempenho, o referencial AQUA atende a dois critérios conforme os indicadores exigidos pela norma de desempenho. Esta subcategoria é atendida pelo AQUA, ainda que deixe de atender a um, dos três critérios exigidos pela norma.

A categoria Conforto acústico apresenta quatro subcategorias, sendo que três delas são contempladas pelo referencial AQUA. Na subcategoria Ruídos de impacto, a norma exige três critérios, onde apenas um é atendido pelo sistema de certificação. Na subcategoria Isolamento dos ruídos entre UH's, a norma estabelece quatro critérios e dois deles já são atendidos pelo referencial AQUA. Na subcategoria Isolamento acústico das vedações, o referencial atende aos dois critérios exigidos pela norma. Esta subcategoria já é atendida pelo sistema de certificação, uma vez que atende aos critérios e indicadores exigidos pela norma NBR 15575.

As categorias Gestão da energia e Gestão da água, embora não apresentem uma preocupação característica da norma de desempenho, ainda assim é contemplada de forma sucinta. Na categoria Gestão da energia, tanto o AQUA quanto a norma apresentam em comum um a recomendação que estabelece indicadores para redução do consumo de energia contemplado em projeto. $\mathrm{Na}$ categoria Gestão da água, o referencial AQUA atende aos indicadores exigidos pela norma para o reuso da água pluvial. No entanto, o controle de qualidade da água não é atendido pelo AQUA. Sendo assim, esta subcategoria é atendida parcialmente pelo sistema de certificação.

Na categoria Gestão da manutenção, o referencial AQUA apresenta em comum com a norma de desempenho a subcategoria Manutenibilidade do edifício e seus sistemas. Os critérios estabelecidos pelo sistema de certificação não tratam dos mesmos temas abordados pela NBR 15575. Considera-se que o AQUA atende parcialmente a norma de desempenho, visto que já estabelece critérios voltados para a mesma preocupação, ainda que não correspondam aos critérios exigidos pela norma.

A categoria Gestão dos resíduos apresenta uma subcategoria em comum aos dois instrumentos de análise. No entanto, o tema é mais peculiar ao sistema de certificação ambiental. Na subcategoria foi identificado um critério em comum, no qual foram estabelecidos os mesmos indicadores por meio de medidas prescritivas.

Após a análise comparativa entre os critérios exigidos pela norma de desempenho e aqueles estabelecidos pelo referencial AQUA, na Tabela 1 apresenta-se a quantificação do total de critérios exigidos pela norma em todas as subcategorias e o total de critérios atendidos pelo sistema de certificação. Os critérios foram quantificados por categorias.

Tabela 1- Quantificação dos critérios da NBR 15575 e do referencial AQUA

\begin{tabular}{l|c|c}
\hline \multirow{2}{*}{ CATEGORIA } & CRITÉRIOS & CRITÉRIOS \\
& EXIGIDOS & ATENDIDOS \\
\cline { 2 - 3 } & NBR 15575 & AQUA \\
\hline PROJETO & $\mathbf{1 2 7}$ & 12 \\
\hline CONFORTO TÉRMICO & $\mathbf{6}$ & 6 \\
\hline CONFORTO LUMÍNICO & $\mathbf{3}$ & 2 \\
\hline CONFORTO ACÚSTICO & $\mathbf{1 0}$ & 5 \\
\hline CONFORTO TÁTIL E ANTROPODINÂMICO & $\mathbf{4}$ & --- \\
\hline
\end{tabular}


/Continuação

\begin{tabular}{l|c|c}
\hline GESTÃO DA ENERGIA & $* * *$ & $* * *$ \\
\hline GESTÃO DA ÁGUA & $\mathbf{1}$ & 0 \\
\hline GESTÃO DA MANUTENÇÃO & $\mathbf{5}$ & 0 \\
\hline GESTÃO DOS RESÍDUOS & $* * *$ & $* * *$ \\
\hline TOTAL & $\mathbf{1 5 6}$ & $\mathbf{2 5}$ \\
\hline
\end{tabular}

Fonte: Elaborado pelas autoras

*** Referente às recomendações, ou seja, não têm o valor de obrigatoriedade de atendimento como os critérios, portanto não foram quantificados para efeito da análise.

A norma NBR 15575 apresenta no total cento e cinqüenta e seis critérios distribuídos nas seis partes que compõem a norma e reorganizados em categorias e subcategorias. De acordo com os resultados apresentados na Tabela 1, o sistema de certificação AQUA já atende a $16 \%$ do total de critérios exigidos pela norma. Dos critérios exigidos pela norma de desempenho e que já são atendidos pelo AQUA nas subcategorias estabelecidas em comum, o sistema de certificação já atende a vinte e cinco dos noventa e cinco critérios exigidos pela NBR 15575. Ou seja, nas subcategorias o AQUA já atende a $26 \%$ dos critérios exigidos pela norma de desempenho.

No contexto geral, o sistema de certificação AQUA já está introduzindo em sua metodologia critérios de desempenho que são exigidos pela norma brasileira de desempenho. Além das exigências de sustentabilidade, os critérios adotados pelo AQUA poderão conferir maior qualidade às unidades geradas. No entanto, as construtoras que já adotam o referencial AQUA ainda terão que atender as demais exigências da norma NBR 15575, uma vez que esta deve ser atendida integralmente.

\section{CONSIDERAÇÕES FINAIS}

A norma brasileira de desempenho, após diversas discussões e consultas públicas entrou em vigor em julho de 2013. Sua aplicação ainda é alvo de discussões, uma vez que a norma ainda é recente. A obrigatoriedade de aplicação da norma é uma importante iniciativa para garantir que as construções de empreendimentos habitacionais ofereçam o desempenho mínimo esperado para atender as necessidades dos usuários durante a vida útil da edificação.

As construtoras e empresas de projeto que já adotam o sistema de certificação ambiental AQUA começam a introduzir durante a fase de projeto os conceitos de desempenho na construção civil. Isto porque o Referencial Técnico para certificação de Edifícios Habitacionais (RT), na versão atualizada em 2013 incorporou exigências estabelecidas pela norma de desempenho. Com a introdução destas exigências na nova versão do referencial, a certificação deverá garantir o melhor desempenho das habitações ao atender as exigências conforme recomenda a norma.

Os resultados das análises identificaram que alguns critérios de desempenho exigidos pela referida norma já estão sendo atendidos pelo AQUA. Outras exigências deixaram de ser atendidas, seja pelo fato de que o sistema de certificação está orientado principalmente para uma avaliação ambiental, seja pelo fato de que a norma ainda é recente. Contudo, a introdução dos critérios de desempenho por meio do sistema de certificação aponta que o conceito de desempenho já está sendo incorporado, ainda que aos poucos, na forma de projetar e construir no Brasil. Esse é mais um importante instrumento para promover condições mínimas de habitabilidade, conforto e qualidade das construções habitacionais no país. 


\section{REFERÊNCIAS}

ASSOCIAÇÃO BRASILEIRA DE NORMAS TÉCNICAS - ABNT - NBR 15.575-1. Edificações habitacionais - Desempenho. Parte 1: Requisitos gerais. São Paulo, 2013. 71p.

NBR 15.575-2: Edificações habitacionais - Desempenho. Parte 2: Requisitos para os sistemas estruturais. São Paulo, 2013. 31p.

NBR 15.575-3: Edificações habitacionais - Desempenho. Parte 3: Requisitos para os sistemas de pisos. São Paulo, 2013. 42p.

NBR 15.575-4: Edificações habitacionais - Desempenho. Parte 4: Requisitos para os sistemas de vedações verticais internas e externas - SVVIE. São Paulo, 2013. 63p.

NBR 15.575-5: Edificações habitacionais - Desempenho. Parte 5: Requisitos para os sistemas de coberturas. São Paulo, 2013. 73p.

NBR 15.575-6: Edificações habitacionais - Desempenho. Parte 6: Requisitos para os sistemas hidrossanitários. São Paulo, 2013. 32p.

BARATELLA, P.R.M. Análise do desenvolvimento de indicadores para a avaliação de sustentabilidade de edifícios brasileiros. 2011. Dissertação (Mestrado em Engenharia Civil) Faculdade de Engenharia Civil, Universidade Estadual de Campinas, Campinas, 2011.

BORGES, C.A.M.; SABBATINI, F.H. O conceito de desempenho de edificações e a sua importância para o setor da construção civil no Brasil. Boletim Técnico da Escola Politécnica da USP, Departamento de Engenharia de Construção Civil, BT/PCC/515. São Paulo: UPUSP, 2008.

FUNDAÇÃO VANZOLINI - FCAV. Referencial Técnico de Certificação - Edifícios Habitacionais. Processo AQUA. Fundação Vanzolini e CERQUAL. 2013. Versão 2.

INSTITUTO DE PESQUISAS TECNOLÓGICAS DO ESTADO DE SÃO PAULO (IPT). Critérios mínimos de desempenho para habitações térreas de interesse social. São Paulo: IPT, 1998, 84p.

INTERNATIONAL ORGANIZATION FOR STANDARDIZATION. ISO 6240: Performance standards in buildings: contents and presentation. London, 1980. 2p.

INTERNATIONAL ORGANIZATION FOR STANDARDIZATION. ISO 6241: Performance standards in buildings: principles for their preparation. London, 1984. 12p.

MITIDIERI FILHO, C.V.; HELENE, P.R.L. Avaliação de desempenho de componentes e elementos construtivos destinados a habitações. Proposições específicas à avaliação do desempenho estrutural. São Paulo: EPUSP, 1998.

OLIVEIRA, A.L.; MITIDIERI FILHO, C.V. O projeto de edifícios habitacionais considerando a Norma Brasileira de Desempenho: análise aplicada para as vedações verticais. Gestão e Tecnologia de Projetos, São Carlos, v.7, n.1, p 90-100, Maio, 2012.

SILVA, V.G. Metodologias de avaliação de desempenho ambiental de edifícios: estado atual e discussão metodológica. Finep 2386/04. São Paulo, 2007.

ROQUE, J.A. O desempenho quanto à durabilidade de alvenarias de blocos cerâmicos de vedação com função auto-portante: o caso da Habitação de Interesse Social. 2009. 212 p. Tese (Doutorado em Engenharia Civil) - Faculdade de Engenharia Civil, Universidade Estadual de Campinas, Campinas, 2009. 